Supporting Information

\title{
syn-(Me,Me)Bimane as a Structural Building Block in Metal Coordination Architectures
}

\author{
Yael Diskin-Posner, ${ }^{\mathrm{a}, *}$ Sara Amer,,${ }^{\mathrm{b}, \dagger}$ Ankana Roy, ${ }^{\mathrm{b}, \uparrow}$ \\ Partha Jyoti Das, ${ }^{\mathrm{b}}$ Flavio Grynszpan, ${ }^{\mathrm{b}}$ Michael Montagb,*
}

${ }^{a}$ Dr. Y. Diskin-Posner

Department of Chemical Research Support

Weizmann Institute of Science

Rehovot 76100, Israel

E-mail: yael.diskin-posner@weizmann.ac.il

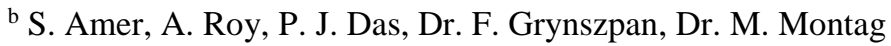

Department of Chemical Sciences

Ariel University

Ariel 40700, Israel

E-mail: michaelmo@ariel.ac.il

$\dagger$ These authors contributed equally. 
Table S1. Crystallographic data for complexes 6-12.

\begin{tabular}{|c|c|c|c|c|c|c|}
\hline Species & 6 & 7 & 8 & $9+10 *$ & 11 & 12 \\
\hline $\mathrm{CCDC}$ & 1879718 & 1879148 & 1879145 & 1879147 & 1879139 & 1879140 \\
\hline Formula & $\mathrm{C}_{20} \mathrm{H}_{28} \mathrm{ClLiN}_{4} \mathrm{O}_{10}$ & $\mathrm{C}_{40} \mathrm{H}_{48} \mathrm{I}_{6} \mathrm{Li}_{2} \mathrm{~N}_{8} \mathrm{O}_{8}$ & $\mathrm{C}_{30} \mathrm{H}_{42} \mathrm{I}_{3} \mathrm{LiN}_{6} \mathrm{O}_{9}$ & $\mathrm{C}_{100} \mathrm{H}_{132} \mathrm{I}_{12} \mathrm{Li}_{4} \mathrm{~N}_{20} \mathrm{O}_{26}$ & $\mathrm{C}_{11} \mathrm{H}_{12} \mathrm{~F}_{3} \mathrm{LiN}_{2} \mathrm{O}_{5} \mathrm{~S}$ & $\mathrm{C}_{10} \mathrm{H}_{12} \mathrm{BF}_{4} \mathrm{LiN}_{2} \mathrm{O}_{2}$ \\
\hline Formula weight $\left(\mathrm{g} \mathrm{mol}^{-1}\right)$ & 526.85 & 1544.14 & 1018.33 & 3580.81 & 348.23 & 285.97 \\
\hline Crystal system & Triclinic & Triclinic & Monoclinic & Triclinic & Monoclinic & Monoclinic \\
\hline Space group & $P \overline{1}$ & $P \overline{1}$ & $P c$ & $P \overline{1}$ & $P 2{ }_{1} / c$ & $P 2_{1} / c$ \\
\hline Crystal size $(\mu \mathrm{m})$ & $41 \times 34 \times 11$ & $220 \times 100 \times 100$ & $386 \times 206 \times 142$ & $540 \times 154 \times 142$ & $282 \times 158 \times 82$ & $122 \times 68 \times 5$ \\
\hline Crystal color and shape & Yellow cube & Dark orange prism & Orange plate & Orange plate & Light yellow prism & Yellow plate \\
\hline Temperature $(\mathrm{K})$ & 100 & 100 & 100 & 100 & 100 & 100 \\
\hline Wavelength $(\AA)$ & 1.54184 & 0.71073 & 0.71073 & 0.71073 & 1.54184 & 1.54184 \\
\hline $\mathrm{a}(\AA)$ & $10.6299(2)$ & $7.8320(4)$ & $12.2165(8)$ & $12.6756(10)$ & $8.5575(1)$ & $11.6055(3)$ \\
\hline $\mathrm{b}(\AA)$ & $10.7507(2)$ & $11.6656(6)$ & $18.0964(11)$ & $14.0845(10)$ & $17.1697(2)$ & $16.2263(2)$ \\
\hline $\mathrm{c}(\AA)$ & $11.5422(2)$ & $14.8267(7)$ & $17.6180(11)$ & $19.6297(15)$ & $9.9338(1)$ & $14.2634(3)$ \\
\hline$\alpha\left(^{\circ}\right)$ & $92.1460(10)$ & $78.9280(10)$ & 90 & $69.701(2)$ & 90 & 90 \\
\hline$\beta\left(^{\circ}\right)$ & $105.777(2)$ & $79.869(2)$ & $97.102(2)$ & $83.260(3)$ & $96.4110(10)$ & 113.931(3) \\
\hline$\gamma\left({ }^{\circ}\right)$ & $107.499(2)$ & $89.4940(10)$ & 90 & $80.422(3)$ & 90 & 90 \\
\hline Volume $\left(\AA^{3}\right)$ & $1200.34(4)$ & $1308.26(11)$ & $3865.0(4)$ & $3234.3(4)$ & $1450.44(3)$ & $2455.11(10)$ \\
\hline $\mathrm{Z}$ & 2 & 1 & 4 & 1 & 4 & 8 \\
\hline$\rho_{\text {calcd, }}\left(\mathrm{g} \mathrm{cm}^{-3}\right)$ & 1.458 & 1.960 & 1.750 & 1.838 & 1.595 & 1.547 \\
\hline$\mu\left(\mathrm{mm}^{-1}\right)$ & 1.965 & 3.615 & 2.481 & 2.945 & 2.574 & 1.271 \\
\hline Number of reflections (unique) & $13400(5177)$ & $93729(12724)$ & $75632(22846)$ & $190124(24824)$ & $10568(2650)$ & $10912(4480)$ \\
\hline $\mathrm{R}_{\text {int }}$ & 0.0305 & 0.0327 & 0.0537 & 0.0390 & 0.0889 & 0.0831 \\
\hline Completeness to $\theta(\%)$ & 99.8 & 99.9 & 99.8 & 99.8 & 99.9 & 100.0 \\
\hline Data / restraints / parameters & $5177 / 0 / 344$ & $12724 / 0 / 307$ & $22846 / 60 / 878$ & $24824 / 0 / 790$ & $2650 / 0 / 212$ & $4480 / 0 / 479$ \\
\hline Goodness-of-fit on $F^{2}$ & 1.124 & 1.059 & 1.029 & 1.061 & 1.055 & 1.092 \\
\hline Final $R_{I}$ and $w R_{2}$ indices $[I>2 \sigma(I)]$ & $0.0463,0.1220$ & $0.0292,0.0582$ & $0.0781,0.2148$ & $0.0289,0.0636$ & $0.0494,0.1403$ & $0.0815,0.2301$ \\
\hline$R_{l}$ and $w R_{2}$ indices (all data) & $0.0488,0.1236$ & $0.0457,0.0653$ & $0.1203,0.2506$ & $0.0464,0.0734$ & $0.0502,0.1415$ & $0.0862,0.2348$ \\
\hline Largest difference: peak, hole $\left(\mathrm{e} \AA^{-3}\right)$ & $0.424,-0.535$ & $1.650,-1.171$ & $5.145,-2.375$ & $1.512,-0.716$ & $0.346,-0.792$ & $0.651,-0.333$ \\
\hline
\end{tabular}

* Complexes $\mathbf{9}$ and $\mathbf{1 0}$ appeared together in the same crystal structure. 
Table S2. Crystallographic data for complexes 13-17.

\begin{tabular}{|c|c|c|c|c|c|}
\hline Species & 13 & 14 & 15 & 16 & 17 \\
\hline $\mathrm{CCDC}$ & 1879141 & 1879142 & 1879144 & 1879146 & 1879143 \\
\hline Formula & $\mathrm{C}_{22} \mathrm{H}_{24} \mathrm{~F}_{6} \mathrm{~N}_{4} \mathrm{Na}_{2} \mathrm{O}_{10} \mathrm{~S}_{2}$ & $\mathrm{C}_{20} \mathrm{H}_{24} \mathrm{Cl}_{2} \mathrm{~N}_{4} \mathrm{Na}_{2} \mathrm{O}_{12}$ & $\mathrm{C}_{30} \mathrm{H}_{36} \mathrm{I}_{6} \mathrm{~N}_{6} \mathrm{Na}_{2} \mathrm{O}_{6}$ & $\mathrm{C}_{10} \mathrm{H}_{12} \mathrm{ClLiN}_{2} \mathrm{O}_{2}$ & $\mathrm{C}_{80} \mathrm{H}_{104} \mathrm{~B}_{2} \mathrm{~F}_{8} \mathrm{Li}_{2} \mathrm{~N}_{16} \mathrm{O}_{20}$ \\
\hline Formula weight $\left(\mathrm{g} \mathrm{mol}^{-1}\right)$ & 728.55 & 629.31 & 1384.03 & 234.61 & 1797.29 \\
\hline Crystal system & Monoclinic & Monoclinic & Monoclinic & Monoclinic & Tetragonal \\
\hline Space group & $P 2_{1} / n$ & $P 2_{1}$ & $P 2{ }_{1} / c$ & $\mathrm{Cm}$ & $I \overline{4}$ \\
\hline Crystal size $(\mu \mathrm{m})$ & $592 \times 162 \times 77$ & $150 \times 100 \times 50$ & $335 \times 73 \times 34$ & $284 \times 57 \times 18$ & $243 \times 62 \times 56$ \\
\hline Crystal color and shape & Yellow plate & Yellow plate & Orange needle & Yellow plate & Yellow plate \\
\hline Temperature $(\mathrm{K})$ & 100 & 296 & 100 & 100 & 100 \\
\hline Wavelength $(\AA)$ & 0.71073 & 0.71073 & 1.54184 & 1.54184 & 1.54184 \\
\hline $\mathrm{a}(\AA)$ & $12.5460(4)$ & $10.5651(3)$ & $15.1168(6)$ & $9.7748(4)$ & $24.8936(1)$ \\
\hline $\mathrm{b}(\AA)$ & $19.3362(5)$ & $11.0702(3)$ & $25.7898(7)$ & $14.0789(4)$ & $24.8936(1)$ \\
\hline $\mathrm{c}(\AA)$ & $13.1082(5)$ & $11.6015(3)$ & $12.5542(5)$ & $3.9433(1)$ & $6.8268(1)$ \\
\hline$\alpha\left(^{\circ}\right)$ & 90 & 90 & 90 & 90 & 90 \\
\hline$\beta\left(^{\circ}\right)$ & $112.288(4)$ & $97.1730(10)$ & $113.397(5)$ & $99.403(4)$ & 90 \\
\hline$\gamma\left({ }^{\circ}\right)$ & 90 & 90 & 90 & 90 & 90 \\
\hline Volume $\left(\AA^{3}\right)$ & $2942.37(18)$ & $1346.27(6)$ & $4491.9(3)$ & $535.38(3)$ & $4230.51(7)$ \\
\hline $\mathrm{Z}$ & 4 & 2 & 4 & 2 & 2 \\
\hline$\rho_{\text {calcd, }}\left(\mathrm{g} \mathrm{cm}^{-3}\right)$ & 1.645 & 1.552 & 2.047 & 1.455 & 1.411 \\
\hline$\mu\left(\mathrm{mm}^{-1}\right)$ & 0.309 & 0.342 & 33.132 & 3.028 & 0.954 \\
\hline Number of reflections (unique) & $21903(18226)$ & $71778(6439)$ & $18830(18830)$ & $8329(1144)$ & $39419(4581)$ \\
\hline $\mathrm{R}_{\mathrm{int}}$ & 0.0271 & 0.0303 & 0.0161 & 0.0817 & 0.0575 \\
\hline Completeness to $\theta(\%)$ & 99.7 & 99.8 & 100.0 & 100.0 & 99.8 \\
\hline Data / restraints / parameters & $18226 / 0 / 424$ & $6439 / 51 / 439$ & $18830 / 9 / 517$ & $1144 / 2 / 82$ & $4581 / 3 / 339$ \\
\hline Goodness-of-fit on $F^{2}$ & 1.101 & 1.036 & 1.084 & 1.095 & 1.079 \\
\hline Final $R_{1}$ and $w R_{2}$ indices $[I>2 \sigma(I)]$ & $0.0736,0.1974$ & $0.0491,0.1381$ & $0.0776,0.2345$ & $0.0498,0.1296$ & $0.0444,0.1152$ \\
\hline$R_{l}$ and $w R_{2}$ indices (all data) & $0.0943,0.2122$ & $0.0720,0.1622$ & $0.0894,0.2515$ & $0.0501,0.1297$ & $0.0457,0.1165$ \\
\hline Largest difference: peak, hole $\left(\mathrm{e} \AA^{-3}\right)$ & $1.256,-0.622$ & $0.311,-0.402$ & $2.243,-1.915$ & $0.491,-0.382$ & $0.210,-0.228$ \\
\hline
\end{tabular}




\section{Metrical parameters: Hydrogen bonding}

The following tables contain interatomic distances and angles for donor and acceptor atoms involved in hydrogen bonding in complexes $6,8,9,10$, and 17. The parameters were calculated using Platon v. 90316 (for Apple Mac OS). In all tables, "D" denotes a hydrogen bond donor atom, and "A" denotes a hydrogen bond acceptor atom. The atom numbering is as defined in the respective CIF files.

\section{Complex 6}

CCDC number: 1879718

\begin{tabular}{|c|c|c|c|c|c|}
\hline $\begin{array}{c}\text { Donor } \\
\text { atom (D) }\end{array}$ & $\begin{array}{c}\text { Hydrogen } \\
\text { atom }\end{array}$ & $\begin{array}{l}\text { Acceptor } \\
\text { atom (A) }\end{array}$ & $\begin{array}{c}\text { Symmetry } \\
\text { operation }\end{array}$ & $\begin{array}{c}\text { D•••A } \\
\text { distance }(\AA)\end{array}$ & $\angle \mathrm{D}-\mathrm{H}-\mathrm{A}\left({ }^{\circ}\right)$ \\
\hline $\mathrm{O}(5)$ & $\mathrm{H}(5 \mathrm{~A})$ & $\mathrm{O}(6)$ & $\mathrm{x}, \mathrm{y}, \mathrm{z}$ & $2.781(2)$ & $175(3)$ \\
\hline $\mathrm{O}(5)$ & $\mathrm{H}(5 \mathrm{~B})$ & $\mathrm{O}(2)$ & 2-x,1-y,1-z & $3.042(2)$ & 151(3) \\
\hline $\mathrm{O}(6)$ & $\mathrm{H}(6 \mathrm{~A})$ & $\mathrm{O}(7)$ & $\mathrm{x}, \mathrm{y}, \mathrm{z}$ & $2.918(2)$ & 168 \\
\hline $\mathrm{O}(6)$ & $\mathrm{H}(6 \mathrm{~B})$ & $\mathrm{O}(4)$ & 1-x,1-y,1-z & $3.064(2)$ & 155 \\
\hline
\end{tabular}

\section{Complex 8}

CCDC number: 1879145

\begin{tabular}{|c|c|c|c|c|c|}
\hline $\begin{array}{c}\text { Donor } \\
\text { atom (D) }\end{array}$ & $\begin{array}{l}\text { Hydrogen } \\
\text { atom }\end{array}$ & $\begin{array}{l}\text { Acceptor } \\
\text { atom (A) }\end{array}$ & $\begin{array}{l}\text { Symmetry } \\
\text { operation }\end{array}$ & 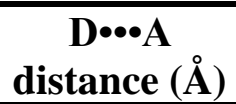 & $\angle$ D-H-A $\left(^{\circ}\right)$ \\
\hline $\mathrm{O}(13)$ & $\mathrm{H}(13 \mathrm{~B})$ & $\mathrm{O}(8)$ & $-1+x, 1-y,-1 / 2+z$ & $2.902(18)$ & 172 \\
\hline $\mathrm{O}(14)$ & $\mathrm{H}(14 \mathrm{~A})$ & $\mathrm{O}(12)$ & $-1+x, 2-y,-1 / 2+z$ & $2.887(15)$ & 156 \\
\hline $\mathrm{O}(14)$ & $\mathrm{H}(14 \mathrm{~B})$ & $\mathrm{O}(16)$ & $-1+x, 1-y,-1 / 2+z$ & $2.940(16)$ & 115 \\
\hline $\mathrm{O}(15)$ & $\mathrm{H}(15 \mathrm{~A})$ & $\mathrm{O}(2)$ & $\mathrm{x}, \mathrm{y}, \mathrm{z}$ & $2.765(17)$ & 162 \\
\hline $\mathrm{O}(15)$ & $\mathrm{H}(15 \mathrm{~B})$ & $\mathrm{O}(17)$ & $-1+x, 1-y,-1 / 2+z$ & $2.936(16)$ & 163 \\
\hline $\mathrm{O}(16)$ & $\mathrm{H}(16 \mathrm{~A})$ & $\mathrm{O}(13)$ & $1+x, 1-y, 1 / 2+z$ & $3.039(18)$ & 116 \\
\hline $\mathrm{O}(16)$ & $\mathrm{H}(16 \mathrm{~B})$ & $\mathrm{O}(4)$ & $\mathrm{x}, \mathrm{y}, \mathrm{z}$ & $2.741(17)$ & 168 \\
\hline $\mathrm{O}(17)$ & $\mathrm{H}(17 \mathrm{~A})$ & $\mathrm{O}(9)$ & $x, 1-y, 1 / 2+z$ & $2.701(16)$ & $157(13)$ \\
\hline $\mathrm{O}(17)$ & $\mathrm{H}(17 \mathrm{~B})$ & $\mathrm{O}(6)$ & $x, 1-y, 1 / 2+z$ & $2.859(15)$ & $165(15)$ \\
\hline $\mathrm{O}(18)$ & $\mathrm{H}(18 \mathrm{~A})$ & $\mathrm{O}(15)$ & $1+x, 1-y, 1 / 2+z$ & $3.126(16)$ & 151 \\
\hline
\end{tabular}

\section{Complexes 9 and 10}

CCDC number: 1879147

\begin{tabular}{|c|c|c|c|c|c|}
\hline $\begin{array}{c}\text { Donor } \\
\text { atom (D) }\end{array}$ & $\begin{array}{c}\text { Hydrogen } \\
\text { atom }\end{array}$ & $\begin{array}{l}\text { Acceptor } \\
\text { atom (A) }\end{array}$ & $\begin{array}{c}\text { Symmetry } \\
\text { operation }\end{array}$ & 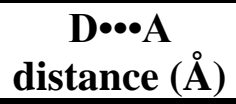 & $\angle \mathrm{D}-\mathrm{H}-\mathrm{A}\left({ }^{\circ}\right)$ \\
\hline $\mathrm{O}(11)$ & $\mathrm{H}(11 \mathrm{~A})$ & $\mathrm{O}(5)$ & $\mathrm{x}, \mathrm{y}, \mathrm{z}$ & $2.840(3)$ & 131 \\
\hline $\mathrm{O}(11)$ & $\mathrm{H}(11 \mathrm{~B})$ & $\mathrm{O}(4)$ & $\mathrm{x}, \mathrm{y}, \mathrm{z}$ & $2.762(3)$ & 170 \\
\hline $\mathrm{O}(12)$ & $\mathrm{H}(12 \mathrm{~A})$ & $\mathrm{O}(10)$ & 1-x,1-y,1-z & $3.005(2)$ & 163 \\
\hline $\mathrm{O}(12)$ & $\mathrm{H}(12 \mathrm{~B})$ & $\mathrm{O}(7)$ & $\mathrm{x}, \mathrm{y}, \mathrm{z}$ & $2.829(2)$ & 170 \\
\hline $\mathrm{O}(13)$ & $\mathrm{H}(13 \mathrm{~A})$ & $\mathrm{O}(9)$ & $\mathrm{x}, \mathrm{y}, \mathrm{z}$ & $2.862(3)$ & 165 \\
\hline $\mathrm{O}(13)$ & $\mathrm{H}(13 \mathrm{~B})$ & $\mathrm{O}(8)$ & 1-x,1-y,1-z & $2.874(2)$ & 174 \\
\hline
\end{tabular}




\section{Complex 17}

CCDC number: 1879143

\begin{tabular}{|c|c|c|c|c|c|}
\hline $\begin{array}{c}\text { Donor } \\
\text { atom (D) }\end{array}$ & $\begin{array}{l}\text { Hydrogen } \\
\text { atom }\end{array}$ & $\begin{array}{l}\text { Acceptor } \\
\text { atom (A) }\end{array}$ & $\begin{array}{l}\text { Symmetry } \\
\text { operation }\end{array}$ & 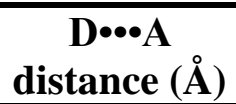 & $\angle \mathrm{D}-\mathrm{H}-\mathrm{A}\left(^{\circ}\right)$ \\
\hline $\mathrm{O}(5)$ & $\mathrm{H}(5 \mathrm{~A})$ & $\mathrm{O}(1)$ & $\mathrm{y}, 1-\mathrm{x}, 1-\mathrm{z}$ & $2.929(3)$ & 158 \\
\hline $\mathrm{O}(5)$ & $\mathrm{H}(5 \mathrm{~B})$ & $\mathrm{O}(1)$ & $\mathrm{x}, \mathrm{y}, \mathrm{z}$ & $2.985(3)$ & 100 \\
\hline $\mathrm{O}(5)$ & $\mathrm{H}(5 \mathrm{~B})$ & $\mathrm{O}(2)$ & $\mathrm{x}, \mathrm{y}, \mathrm{z}$ & $2.858(4)$ & 133 \\
\hline
\end{tabular}

\section{Metrical parameters: $\pi-\pi$ stacking}

The following tables contain pertinent parameters associated with short inter-ring contacts, representing $\pi-\pi$ stacking between molecules of syn-(Me,Me)bimane in complexes $6,7, \mathbf{8 , 9}, 10,11$, and 17. The parameters were calculated using Platon v. 90316 (for Apple Mac OS), and are defined as follows:

- $\mathrm{Cg}(i)$ : ring plane number $i$

- $\alpha$ : dihedral angle between ring planes $i$ and $j$

- $\mathrm{Cg}-\mathrm{Cg}$ : distance between ring centroids

- $\mathrm{Cg}(i) \_$perp: perpendicular distance of $\mathrm{Cg}(i)$ on ring $i$

- $\mathrm{Cg}(j)$ _perp: perpendicular distance of $\mathrm{Cg}(j)$ on ring $j$

- Slippage: distance between $\operatorname{Cg}(i)$ and the perpendicular projection of $\mathrm{Cg}(j)$ on ring $i$.

\section{Complex 6}

CCDC number: 1879718

Relevant ring planes: $\mathrm{Cg}(1)=\mathrm{N} 1-\mathrm{N} 2-\mathrm{C} 3-\mathrm{C} 2-\mathrm{C} 1 ; \mathrm{Cg}(2)=\mathrm{N} 1-\mathrm{N} 2-\mathrm{C} 4-\mathrm{C} 5-\mathrm{C} 6$; $\mathrm{Cg}(3)=\mathrm{N} 3-\mathrm{N} 4-\mathrm{C} 13-\mathrm{C} 12-\mathrm{C} 11 ; \mathrm{Cg}(4)=\mathrm{N} 3-\mathrm{N} 4-\mathrm{C} 14-\mathrm{C} 15-\mathrm{C} 16$

\begin{tabular}{|c|c|c|c|c|c|c|c|}
\hline $\operatorname{Cg}(i)$ & $\operatorname{Cg}(j)$ & $\begin{array}{c}\text { Symmetry } \\
\operatorname{Cg}(j)\end{array}$ & $\operatorname{Cg}-\mathrm{Cg}(\AA)$ & $\alpha\left(^{\circ}\right)$ & $\begin{array}{c}\mathrm{Cg}(i)_{(\bar{\AA})} \text { perp } \\
(\mathrm{A})\end{array}$ & $\begin{array}{c}\mathrm{Cg}(j)_{(\AA)} \text { perp } \\
(\mathrm{A})\end{array}$ & $\begin{array}{c}\text { Slippage } \\
(\AA)\end{array}$ \\
\hline $\operatorname{Cg}(1)$ & $\overline{C g}(1)$ & $2-x, 1-y, 2-z$ & $5.3240(12)$ & $0.05(11)$ & $4.0117(8)$ & $4.0116(8)$ & 3.500 \\
\hline $\mathrm{Cg}(1)$ & $\mathrm{Cg}(2)$ & 2-x,1-y,2-z & $4.4509(12)$ & $13.24(11)$ & $3.4750(8)$ & $3.7916(8)$ & 2.331 \\
\hline $\mathrm{Cg}(1)$ & $\mathrm{Cg}(3)$ & $2-x, 1-y, 1-z$ & $5.7268(12)$ & $13.26(11)$ & $-2.8543(8)$ & $-3.1397(8)$ & 4.789 \\
\hline $\mathrm{Cg}(1)$ & $\mathrm{Cg}(4)$ & $1-x, 1-y, 1-z$ & $5.7251(12)$ & $16.13(11)$ & $-3.0150(8)$ & $-3.9971(8)$ & 4.099 \\
\hline $\mathrm{Cg}(2)$ & $\mathrm{Cg}(1)$ & $2-x, 1-y, 2-z$ & $4.4509(12)$ & $13.24(11)$ & $3.7915(8)$ & $3.4750(8)$ & 2.781 \\
\hline $\mathrm{Cg}(2)$ & $\mathrm{Cg}(2)$ & $2-x, 1-y, 2-z$ & $4.2972(12)$ & $0.02(11)$ & $3.6917(8)$ & $3.6918(8)$ & 2.199 \\
\hline $\mathrm{Cg}(2)$ & $\mathrm{Cg}(3)$ & $2-x, 1-y, 1-z$ & $4.4780(12)$ & $18.94(11)$ & $-3.0671(8)$ & $-3.9233(8)$ & 2.159 \\
\hline $\mathrm{Cg}(2)$ & $\mathrm{Cg}(4)$ & $2-x, 1-y, 1-z$ & $5.7672(13)$ & $14.56(11)$ & $-3.5885(8)$ & $-4.2073(8)$ & 3.945 \\
\hline $\mathrm{Cg}(3)$ & $\mathrm{Cg}(2)$ & $2-x, 1-y, 1-z$ & $4.4780(12)$ & $18.94(11)$ & $-3.9234(8)$ & $-3.0671(8)$ & 3.263 \\
\hline $\mathrm{Cg}(3)$ & $\mathrm{Cg}(3)$ & 2-x,2-y,1-z & $3.3970(12)$ & $0.00(11)$ & $3.2324(8)$ & $3.2324(8)$ & 1.045 \\
\hline $\mathrm{Cg}(3)$ & $\mathrm{Cg}(4)$ & 2-x,2-y,1-z & $3.4973(12)$ & $8.66(11)$ & $3.2057(8)$ & $3.3758(8)$ & 0.914 \\
\hline $\mathrm{Cg}(4)$ & $\mathrm{Cg}(1)$ & $1-x, 1-y, 1-z$ & $5.7252(12)$ & $16.13(11)$ & $-3.9972(8)$ & $-3.0150(8)$ & 4.867 \\
\hline $\mathrm{Cg}(4)$ & $\mathrm{Cg}(2)$ & $2-x, 1-y, 1-z$ & $5.7673(13)$ & $14.56(11)$ & $-4.2074(8)$ & $-3.5884(8)$ & 4.515 \\
\hline $\mathrm{Cg}(4)$ & $\operatorname{Cg}(3)$ & $2-x, 2-y, 1-z$ & $3.4973(12)$ & $8.66(11)$ & $3.3757(8)$ & $3.2057(8)$ & 1.398 \\
\hline $\mathrm{Cg}(4)$ & $\mathrm{Cg}(4)$ & $2-x, 2-y, 1-z$ & $4.4847(12)$ & $0.02(11)$ & $3.0627(8)$ & $3.0628(8)$ & 3.276 \\
\hline
\end{tabular}




\section{Complex 7}

CCDC number: 1879148

Relevant ring planes: $\mathrm{Cg}(1)=\mathrm{N} 1-\mathrm{N} 2-\mathrm{C} 3-\mathrm{C} 2-\mathrm{C} 1 ; \mathrm{Cg}(2)=\mathrm{N} 1-\mathrm{N} 2-\mathrm{C} 4-\mathrm{C} 5-\mathrm{C} 6$;

$\mathrm{Cg}(3)=\mathrm{N} 3-\mathrm{N} 4-\mathrm{C} 13-\mathrm{C} 12-\mathrm{C} 11 ; \mathrm{Cg}(4)=\mathrm{N} 3-\mathrm{N} 4-\mathrm{C} 14-\mathrm{C} 15-\mathrm{C} 16$

\begin{tabular}{|c|c|c|c|c|c|c|c|}
\hline $\operatorname{Cg}(i)$ & $\operatorname{Cg}(j)$ & $\begin{array}{c}\text { Symmetry } \\
\mathrm{Cg}(j)\end{array}$ & $\mathrm{Cg}-\mathrm{Cg}(\AA)$ & $\alpha\left(^{\circ}\right)$ & $\begin{array}{c}\mathrm{Cg}(i) \_ \text {perp } \\
(\mathrm{A})\end{array}$ & $\underset{(\mathbf{A})}{\operatorname{Cg}(j)_{-} \text {perp }}$ & $\begin{array}{c}\text { Slippage } \\
(\AA)\end{array}$ \\
\hline $\operatorname{Cg}(3)$ & $\mathrm{Cg}(3)$ & 2-x,2-y,1-z & $3.5341(13)$ & $0.03(13)$ & $3.2593(9)$ & $3.2594(9)$ & 1.366 \\
\hline $\operatorname{Cg}(3)$ & $\mathrm{Cg}(4)$ & $2-x, 2-y, 1-z$ & $3.5135(13)$ & $7.43(13)$ & $3.2529(9)$ & $3.3832(9)$ & 0.948 \\
\hline $\operatorname{Cg}(4)$ & $\mathrm{Cg}(3)$ & $2-x, 2-y, 1-z$ & $3.5136(13)$ & $7.43(13)$ & $3.3832(9)$ & $3.2529(9)$ & 1.328 \\
\hline $\operatorname{Cg}(4)$ & $\mathrm{Cg}(4)$ & $2-x, 2-y, 1-z$ & $4.4083(13)$ & $0.02(13)$ & $3.1336(10)$ & $3.1336(10)$ & 3.101 \\
\hline
\end{tabular}

\section{Complex 8}

CCDC number: 1879145

Relevant ring planes: $\mathrm{Cg}(1)=\mathrm{N} 1-\mathrm{N} 2-\mathrm{C} 3-\mathrm{C} 2-\mathrm{C} 1 ; \mathrm{Cg}(2)=\mathrm{N} 1-\mathrm{N} 2-\mathrm{C} 4-\mathrm{C} 5-\mathrm{C} 6$;

$\mathrm{Cg}(3)=\mathrm{N} 3-\mathrm{N} 4-\mathrm{C} 13-\mathrm{C} 12-\mathrm{C} 11 ; \mathrm{Cg}(4)=\mathrm{N} 3-\mathrm{N} 4-\mathrm{C} 14-\mathrm{C} 15-\mathrm{C} 16$;

$\mathrm{Cg}(5)=\mathrm{N} 5-\mathrm{N} 6-\mathrm{C} 23-\mathrm{C} 22-\mathrm{C} 21 ; \mathrm{Cg}(6)=\mathrm{N} 5-\mathrm{N} 6-\mathrm{C} 24-\mathrm{C} 25-\mathrm{C} 26$;

$\mathrm{Cg}(7)=\mathrm{N} 7-\mathrm{N} 8-\mathrm{C} 33-\mathrm{C} 32-\mathrm{C} 31 ; \mathrm{Cg}(8)=\mathrm{N} 7-\mathrm{N} 8-\mathrm{C} 34-35-\mathrm{C} 36$;

$\mathrm{Cg}(9)=\mathrm{N} 9-\mathrm{N} 10-\mathrm{C} 43-\mathrm{C} 42-\mathrm{C} 41 ; \mathrm{Cg}(10)=\mathrm{N} 9-\mathrm{N} 10-\mathrm{C} 44-\mathrm{C} 45-\mathrm{C} 46$;

$\mathrm{Cg}(11)=\mathrm{N} 11-\mathrm{N} 12-\mathrm{C} 53-\mathrm{C} 52-\mathrm{C} 51 ; \mathrm{Cg}(12)=\mathrm{N} 11-\mathrm{N} 12-\mathrm{C} 54-\mathrm{C} 55-\mathrm{C} 56$

\begin{tabular}{|c|c|c|c|c|c|c|c|}
\hline $\operatorname{Cg}(i)$ & $\operatorname{Cg}(j)$ & $\begin{array}{c}\text { Symmetry } \\
\mathrm{Cg}(j)\end{array}$ & $\operatorname{Cg}-\mathrm{Cg}(\AA)$ & $\alpha\left({ }^{\circ}\right)$ & $\begin{array}{c}\mathrm{Cg}(i) \_ \text {perp } \\
(\AA \mathbf{\AA})\end{array}$ & $\begin{array}{c}\mathrm{Cg}(j)_{\bar{\AA}} \text { perp } \\
(\mathbf{\AA})\end{array}$ & $\begin{array}{c}\text { Slippage } \\
\text { (A) }\end{array}$ \\
\hline $\operatorname{Cg}(1)$ & $\operatorname{Cg}(3)$ & $x, 1-y,-1 / 2+z$ & $3.617(10)$ & 2 & $3.074(7)$ & $3.140(7)$ & 1.794 \\
\hline $\mathrm{Cg}(1)$ & $\mathrm{Cg}(4)$ & $x, 1-y,-1 / 2+z$ & $3.349(10)$ & 8 & $3.159(7)$ & $3.234(7)$ & 0.871 \\
\hline $\mathrm{Cg}(2)$ & $\mathrm{Cg}(3)$ & $x, 1-y,-1 / 2+z$ & $3.361(10)$ & 8 & $3.251(7)$ & $3.191(7)$ & 1.054 \\
\hline $\mathrm{Cg}(2)$ & $\mathrm{Cg}(4)$ & $x, 1-y,-1 / 2+z$ & $4.082(10)$ & 2 & $2.985(6)$ & $3.091(7)$ & 2.666 \\
\hline $\mathrm{Cg}(3)$ & $\mathrm{Cg}(1)$ & $x, 1-y, 1 / 2+z$ & $3.618(10)$ & 8 & $3.141(7)$ & $3.075(7)$ & 1.906 \\
\hline $\mathrm{Cg}(3)$ & $\mathrm{Cg}(2)$ & $x, 1-y, 1 / 2+z$ & $3.362(10)$ & 8 & $3.192(7)$ & $3.251(7)$ & 0.853 \\
\hline $\mathrm{Cg}(4)$ & $\mathrm{Cg}(1)$ & $x, 1-y, 1 / 2+z$ & $3.349(10)$ & 2 & $3.234(7)$ & $3.159(7)$ & 1.113 \\
\hline $\mathrm{Cg}(4)$ & $\mathrm{Cg}(2)$ & $x, 1-y, 1 / 2+z$ & $4.082(10)$ & 13 & $3.091(7)$ & $2.985(6)$ & 2.783 \\
\hline $\mathrm{Cg}(5)$ & $\operatorname{Cg}(7)$ & $\mathrm{x}, \mathrm{y}, \mathrm{z}$ & $3.543(11)$ & 6 & $-3.285(8)$ & $3.311(8)$ & 1.262 \\
\hline $\operatorname{Cg}(5)$ & $\mathrm{Cg}(8)$ & $\mathrm{x}, \mathrm{y}, \mathrm{z}$ & $4.189(11)$ & 9 & $-2.877(8)$ & $3.177(8)$ & 2.731 \\
\hline $\operatorname{Cg}(6)$ & $\operatorname{Cg}(7)$ & $\mathrm{x}, \mathrm{y}, \mathrm{z}$ & $3.734(10)$ & 10 & $-2.945(7)$ & $3.299(8)$ & 1.747 \\
\hline $\operatorname{Cg}(6)$ & $\mathrm{Cg}(8)$ & $\mathrm{x}, \mathrm{y}, \mathrm{z}$ & $3.425(11)$ & 9 & $-3.131(7)$ & $3.286(8)$ & 0.964 \\
\hline $\operatorname{Cg}(7)$ & $\mathrm{Cg}(5)$ & $\mathrm{x}, \mathrm{y}, \mathrm{z}$ & $3.543(11)$ & 13 & $3.311(8)$ & $-3.285(8)$ & 1.326 \\
\hline $\mathrm{Cg}(7)$ & $\mathrm{Cg}(6)$ & $\mathrm{x}, \mathrm{y}, \mathrm{z}$ & $3.733(10)$ & 10 & $3.299(8)$ & $-2.946(7)$ & 2.292 \\
\hline $\mathrm{Cg}(8)$ & $\mathrm{Cg}(5)$ & $\mathrm{x}, \mathrm{y}, \mathrm{z}$ & $4.191(11)$ & 6 & $3.178(8)$ & $-2.877(8)$ & 3.047 \\
\hline $\mathrm{Cg}(8)$ & $\mathrm{Cg}(6)$ & $\mathrm{x}, \mathrm{y}, \mathrm{z}$ & $3.425(11)$ & 9 & $3.286(8)$ & $-3.131(7)$ & 1.388 \\
\hline $\mathrm{Cg}(9)$ & $\mathrm{Cg}(11)$ & $\mathrm{x}, \mathrm{y}, \mathrm{z}$ & $3.578(11)$ & 15 & $-3.376(7)$ & $3.357(8)$ & 1.238 \\
\hline $\mathrm{Cg}(9)$ & $\mathrm{Cg}(12)$ & $\mathrm{x}, \mathrm{y}, \mathrm{z}$ & $3.767(11)$ & 13 & $-3.375(7)$ & $2.919(8)$ & 2.381 \\
\hline $\mathrm{Cg}(10)$ & $\mathrm{Cg}(11)$ & $\mathrm{x}, \mathrm{y}, \mathrm{z}$ & $4.155(11)$ & 5 & $-3.224(8)$ & $2.965(8)$ & 2.910 \\
\hline $\mathrm{Cg}(10)$ & $\mathrm{Cg}(12)$ & $\mathrm{x}, \mathrm{y}, \mathrm{z}$ & $3.406(12)$ & 8 & $-3.286(8)$ & $3.160(8)$ & 1.271 \\
\hline $\mathrm{Cg}(11)$ & $\mathrm{Cg}(9)$ & $\mathrm{x}, \mathrm{y}, \mathrm{z}$ & $3.578(11)$ & 15 & $3.357(8)$ & $-3.376(7)$ & 1.186 \\
\hline $\mathrm{Cg}(11)$ & $\mathrm{Cg}(10)$ & $\mathrm{x}, \mathrm{y}, \mathrm{z}$ & $4.154(11)$ & 5 & $2.965(8)$ & $-3.224(8)$ & 2.619 \\
\hline $\mathrm{Cg}(12)$ & $\mathrm{Cg}(9)$ & $\mathrm{x}, \mathrm{y}, \mathrm{z}$ & $3.766(11)$ & 13 & $2.919(8)$ & $-3.375(7)$ & 1.673 \\
\hline $\mathrm{Cg}(12)$ & $\mathrm{Cg}(10)$ & $\mathrm{x}, \mathrm{y}, \mathrm{z}$ & $3.405(12)$ & 8 & $3.159(8)$ & $-3.286(8)$ & 0.894 \\
\hline
\end{tabular}




\section{Complexes 9 and 10}

CCDC number: 1879147

Relevant ring planes: $\mathrm{Cg}(1)=\mathrm{N} 5-\mathrm{N} 6-\mathrm{C} 23-\mathrm{C} 22-\mathrm{C} 21 ; \mathrm{Cg}(2)=\mathrm{N} 5-\mathrm{N} 6-\mathrm{C} 24-\mathrm{C} 25-\mathrm{C} 26$;

$\mathrm{Cg}(3)=\mathrm{N} 1-\mathrm{N} 2-\mathrm{C} 3-\mathrm{C} 2-\mathrm{C} 1 ; \mathrm{Cg}(4)=\mathrm{N} 7-\mathrm{N} 8-\mathrm{C} 34-35-\mathrm{C} 36 ; \mathrm{Cg}(5)=\mathrm{N} 3-\mathrm{N} 4-\mathrm{C} 13-\mathrm{C} 12-\mathrm{C} 11 ; \mathrm{Cg}(6)=$ N3-N4-C14-C15-C16; Cg(7) = N7-N8-C33-C32-C31; Cg(8) = N7-N8-C34-35-C36; Cg(9) = N9$\mathrm{N} 10-\mathrm{C} 43-\mathrm{C} 42-\mathrm{C} 41 ; \mathrm{Cg}(10)=\mathrm{N} 9-\mathrm{N} 10-\mathrm{C} 44-\mathrm{C} 45-\mathrm{C} 46$;

$\mathrm{Cg}(11)=\mathrm{N} 11-\mathrm{N} 12-\mathrm{C} 53-\mathrm{C} 52-\mathrm{C} 51 ; \mathrm{Cg}(12)=\mathrm{N} 11-\mathrm{N} 12-\mathrm{C} 54-\mathrm{C} 55-\mathrm{C} 56$

\begin{tabular}{|c|c|c|c|c|c|c|c|}
\hline $\mathrm{Cg}(i)$ & $\operatorname{Cg}(j)$ & $\begin{array}{c}\text { Symmetry } \\
\mathrm{Cg}(j)\end{array}$ & $\operatorname{Cg}-\mathrm{Cg}(\AA)$ & $\alpha\left(^{\circ}\right)$ & $\begin{array}{c}\mathrm{Cg}(i) \_ \text {perp } \\
(\mathrm{\AA})\end{array}$ & $\begin{array}{c}\mathrm{Cg}(j)_{(\mathrm{\AA})} \text { perp } \\
\end{array}$ & $\begin{array}{c}\text { Slippage } \\
\text { (Å) }\end{array}$ \\
\hline $\mathrm{Cg}(1)$ & $\operatorname{Cg}(7)$ & $1-x, 1-y, 1-z$ & $3.9733(15)$ & $26.57(14)$ & $3.7632(10)$ & $3.0929(10)$ & - \\
\hline $\mathrm{Cg}(1)$ & $\operatorname{Cg}(8)$ & $1-x, 1-y, 1-z$ & $3.6258(14)$ & $13.16(13)$ & $3.5233(10)$ & $3.5643(10)$ & 0.665 \\
\hline $\mathrm{Cg}(1)$ & $\mathrm{Cg}(9)$ & $1-x, 1-y, 1-z$ & $4.1757(13)$ & $31.47(13)$ & $-2.7332(10)$ & $-3.9769(9)$ & - \\
\hline $\mathrm{Cg}(1)$ & $\mathrm{Cg}(10)$ & $1-x, 1-y, 1-z$ & $3.6958(14)$ & $13.56(12)$ & $3.2573(9)$ & $-3.5498(9)$ & 1.028 \\
\hline $\mathrm{Cg}(2)$ & $\operatorname{Cg}(7)$ & $1-x, 1-y, 1-z$ & $3.5832(14)$ & $11.69(14)$ & $3.4133(10)$ & $3.4509(11)$ & 0.965 \\
\hline $\mathrm{Cg}(2)$ & $\mathrm{Cg}(8)$ & $1-x, 1-y, 1-z$ & $4.1560(14)$ & $24.56(13)$ & $3.7842(9)$ & $3.0593(10)$ & - \\
\hline $\mathrm{Cg}(2)$ & $\mathrm{Cg}(9)$ & $1-x, 1-y, 1-z$ & $3.6861(14)$ & $12.32(13)$ & $-3.2414(10)$ & $-3.5064(9)$ & 1.137 \\
\hline $\mathrm{Cg}(2)$ & $\mathrm{Cg}(10)$ & $1-x, 1-y, 1-z$ & $4.0955(14)$ & $31.11(13)$ & $2.7818(9)$ & $-3.9288(9)$ & - \\
\hline $\operatorname{Cg}(5)$ & $\operatorname{Cg}(5)$ & $2-x,-y, 1-z$ & $4.9364(13)$ & $0.00(13)$ & $3.8440(9)$ & $3.8440(9)$ & 3.097 \\
\hline $\operatorname{Cg}(5)$ & $\operatorname{Cg}(6)$ & $2-x,-y, 1-z$ & $5.1206(14)$ & $24.86(13)$ & $-4.0192(10)$ & $3.4429(9)$ & - \\
\hline $\mathrm{Cg}(5)$ & $\mathrm{Cg}(9)$ & 2-x,1-y,1-z & $4.2395(14)$ & $33.69(13)$ & $4.1420(10)$ & $-2.9751(9)$ & - \\
\hline $\mathrm{Cg}(5)$ & $\mathrm{Cg}(10)$ & 2-x,1-y,1-z & $3.7727(13)$ & $17.50(12)$ & $-3.6122(9)$ & $-3.4018(9)$ & 1.631 \\
\hline $\mathrm{Cg}(6)$ & $\mathrm{Cg}(5)$ & $2-x,-y, 1-z$ & $5.1207(14)$ & $24.86(13)$ & $3.4430(9)$ & $-4.0192(10)$ & - \\
\hline $\mathrm{Cg}(6)$ & $\mathrm{Cg}(6)$ & $2-x,-y, 1-z$ & & & $-4.4256(10)$ & $-4.4256(10)$ & 3.941 \\
\hline $\mathrm{Cg}(6)$ & $\mathrm{Cg}(9)$ & $2-x, 1-y, 1-z$ & $3.5887(14)$ & $8.85(13)$ & $3.4589(10)$ & $3.5582(9)$ & 0.467 \\
\hline $\operatorname{Cg}(6)$ & $\operatorname{Cg}(10)$ & $2-x, 1-y, 1-z$ & $3.8746(13)$ & $23.81(13)$ & $-3.7639(9)$ & $3.2991(9)$ & - \\
\hline $\operatorname{Cg}(7)$ & $\mathrm{Cg}(1)$ & $1-x, 1-y, 1-z$ & $3.9733(15)$ & $26.57(14)$ & $3.0929(10)$ & $3.7632(10)$ & - \\
\hline $\operatorname{Cg}(7)$ & $\mathrm{Cg}(2)$ & 1-x,1-y,1-z & $3.5833(14)$ & $11.69(14)$ & $3.4509(11)$ & $3.4134(10)$ & 1.090 \\
\hline $\mathrm{Cg}(7)$ & $\mathrm{Cg}(3)$ & $1-x, 1-y, 1-z$ & $5.8625(15)$ & $81.77(14)$ & $-0.8374(10)$ & $-5.8603(10)$ & - \\
\hline $\mathrm{Cg}(8)$ & $\mathrm{Cg}(1)$ & 1-x,1-y,1-z & $3.6259(14)$ & $13.16(13)$ & $3.5644(10)$ & $3.5232(10)$ & 0.857 \\
\hline $\mathrm{Cg}(8)$ & $\mathrm{Cg}(2)$ & $1-x, 1-y, 1-z$ & $4.1561(14)$ & $24.56(13)$ & $3.0594(10)$ & $3.7843(9)$ & - \\
\hline $\mathrm{Cg}(9)$ & $\mathrm{Cg}(1)$ & $1-x, 1-y, 1-z$ & $4.1757(13)$ & $31.47(13)$ & $-3.9769(9)$ & $-2.7332(10)$ & - \\
\hline $\mathrm{Cg}(9)$ & $\mathrm{Cg}(2)$ & $1-x, 1-y, 1-z$ & $3.6861(14)$ & $12.32(13)$ & $-3.5063(9)$ & $-3.2413(10)$ & 1.755 \\
\hline $\mathrm{Cg}(9)$ & $\operatorname{Cg}(5)$ & $2-x, 1-y, 1-z$ & $4.2395(14)$ & $33.69(13)$ & $-2.9750(9)$ & $4.1420(10)$ & - \\
\hline $\mathrm{Cg}(9)$ & $\operatorname{Cg}(6)$ & $2-x, 1-y, 1-z$ & $3.5887(14)$ & $8.85(13)$ & $3.5582(9)$ & $3.4588(10)$ & 0.957 \\
\hline $\mathrm{Cg}(10)$ & $\mathrm{Cg}(1)$ & $1-x, 1-y, 1-z$ & $3.6958(14)$ & $13.56(12)$ & $-3.5499(9)$ & $3.2573(9)$ & 1.746 \\
\hline $\mathrm{Cg}(10)$ & $\mathrm{Cg}(2)$ & $1-x, 1-y, 1-z$ & $4.0954(14)$ & $31.11(13)$ & $-3.9289(9)$ & $2.7817(9)$ & - \\
\hline $\mathrm{Cg}(10)$ & $\operatorname{Cg}(5)$ & $2-x, 1-y, 1-z$ & $3.7726(13)$ & $17.50(12)$ & $-3.4017(9)$ & $-3.6121(9)$ & 1.088 \\
\hline $\mathrm{Cg}(10)$ & $\operatorname{Cg}(6)$ & $2-x, 1-y, 1-z$ & $3.8746(13)$ & $23.81(13)$ & $3.2991(9)$ & $-3.7639(9)$ & - \\
\hline
\end{tabular}




\section{Complex 11}

CCDC number: 1879139

Relevant ring planes: $\mathrm{Cg}(1)=\mathrm{N} 1-\mathrm{N} 2-\mathrm{C} 3-\mathrm{C} 2-\mathrm{C} 1 ; \mathrm{Cg}(2)=\mathrm{N} 1-\mathrm{N} 2-\mathrm{C} 4-\mathrm{C} 5-\mathrm{C} 6$

\begin{tabular}{|c|c|c|c|c|c|c|c|}
\hline $\operatorname{Cg}(i)$ & $\mathrm{Cg}(j)$ & $\begin{array}{c}\text { Symmetry } \\
\mathrm{Cg}(j)\end{array}$ & $\mathrm{Cg}-\mathrm{Cg}(\AA)$ & $\alpha\left(^{\circ}\right)$ & $\begin{array}{c}\mathrm{Cg}(i) \_ \text {perp } \\
(\AA)\end{array}$ & $\begin{array}{c}\mathrm{Cg}(j)_{\bar{\delta}} \text { perp } \\
(\AA)\end{array}$ & $\begin{array}{c}\text { Slippage } \\
\text { (A) }\end{array}$ \\
\hline $\mathrm{Cg}(1)$ & $\mathrm{Cg}(1)$ & $2-x, 1-y, 1-z$ & $3.4523(13)$ & $0.00(13)$ & $3.4462(9)$ & $3.4463(9)$ & 0.203 \\
\hline $\operatorname{Cg}(1)$ & $\mathrm{Cg}(2)$ & $2-x, 1-y, 1-z$ & $3.8182(14)$ & $3.57(13)$ & $3.4886(10)$ & $3.3860(9)$ & 1.764 \\
\hline $\mathrm{Cg}(2)$ & $\mathrm{Cg}(1)$ & $2-x, 1-y, 1-z$ & $3.8181(14)$ & $3.57(13)$ & $3.3859(9)$ & $3.4886(10)$ & 1.552 \\
\hline $\mathrm{Cg}(2)$ & $\mathrm{Cg}(2)$ & $2-x, 1-y, 1-z$ & $4.9507(13)$ & $0.00(13)$ & $3.5449(10)$ & $3.5450(10)$ & 3.456 \\
\hline
\end{tabular}

\section{Complex 16}

CCDC number: 1879146

Relevant ring planes: $\mathrm{Cg}(1)=\mathrm{N} 1-\mathrm{N} 2-\mathrm{C} 3-\mathrm{C} 2-\mathrm{C} 1 ; \mathrm{Cg}(2)=\mathrm{N} 1-\mathrm{N} 2-\mathrm{C} 3 *-\mathrm{C} 2 *_{-} \mathrm{C} 1 *$

(* These atoms are symmetry-generated at $\mathrm{x},-\mathrm{y}, \mathrm{z}$ )

\begin{tabular}{|c|c|c|c|c|c|c|c|}
\hline $\operatorname{Cg}(i)$ & $\operatorname{Cg}(j)$ & $\begin{array}{c}\text { Symmetry } \\
\operatorname{Cg}(j)\end{array}$ & $\operatorname{Cg}-\operatorname{Cg}(\AA)$ & $\alpha\left(^{\circ}\right)$ & $\begin{array}{c}\mathrm{Cg}(i)_{(\bar{\AA}} \text { perp } \\
(\mathrm{A})\end{array}$ & $\begin{array}{c}\mathrm{Cg}(j)_{(\AA)} \text { perp } \\
(\mathrm{A})\end{array}$ & $\begin{array}{c}\text { Slippage } \\
\text { (Å) }\end{array}$ \\
\hline $\mathrm{Cg}(1)$ & $\mathrm{Cg}(1)$ & $\mathrm{x}, \mathrm{y},-1+\mathrm{z}$ & $3.943(4)$ & $0.0(3)$ & $3.378(2)$ & $-3.379(2)$ & 2.034 \\
\hline $\mathrm{Cg}(1)$ & $\operatorname{Cg}(1)$ & $\mathrm{x}, \mathrm{y}, 1+\mathrm{z}$ & $3.943(4)$ & $0.0(3)$ & $-3.379(2)$ & $3.378(2)$ & 2.034 \\
\hline $\operatorname{Cg}(1)$ & $\mathrm{Cg}(1)$ & $x,-y,-1+z$ & $4.357(3)$ & $32.6(3)$ & $2.858(2)$ & $-3.898(2)$ & - \\
\hline $\mathrm{Cg}(1)$ & $\mathrm{Cg}(1)$ & $\mathrm{x},-\mathrm{y}, 1+\mathrm{z}$ & $4.357(3)$ & $32.6(3)$ & $-3.898(2)$ & $2.858(2)$ & - \\
\hline $\mathrm{Cg}(1)$ & $\mathrm{Cg}(2)$ & $\mathrm{x}, \mathrm{y},-1+\mathrm{z}$ & $4.357(3)$ & $32.6(3)$ & $2.858(2)$ & $-3.898(2)$ & - \\
\hline $\operatorname{Cg}(1)$ & $\mathrm{Cg}(2)$ & $\mathrm{x}, \mathrm{y}, 1+\mathrm{z}$ & $4.357(3)$ & $32.6(3)$ & $-3.898(2)$ & $2.858(2)$ & - \\
\hline $\mathrm{Cg}(1)$ & $\mathrm{Cg}(2)$ & $\mathrm{x},-\mathrm{y},-1+\mathrm{z}$ & $3.943(4)$ & $0.0(3)$ & $3.378(2)$ & $-3.379(2)$ & 2.034 \\
\hline $\mathrm{Cg}(1)$ & $\mathrm{Cg}(2)$ & $\mathrm{x},-\mathrm{y}, 1+\mathrm{z}$ & $3.943(4)$ & $0.0(3)$ & $-3.379(2)$ & $3.378(2)$ & 2.034 \\
\hline $\operatorname{Cg}(2)$ & $\mathrm{Cg}(1)$ & $\mathrm{x}, \mathrm{y},-1+\mathrm{z}$ & $4.357(3)$ & $32.6(3)$ & $2.858(2)$ & $-3.898(2)$ & - \\
\hline $\operatorname{Cg}(2)$ & $\operatorname{Cg}(1)$ & $\mathrm{x}, \mathrm{y}, 1+\mathrm{z}$ & $4.357(3)$ & $32.6(3)$ & $-3.898(2)$ & $2.858(2)$ & - \\
\hline $\operatorname{Cg}(2)$ & $\mathrm{Cg}(1)$ & $x,-y,-1+z$ & $3.943(4)$ & $0.0(3)$ & $3.378(2)$ & $-3.379(2)$ & 2.034 \\
\hline $\operatorname{Cg}(2)$ & $\mathrm{Cg}(1)$ & $\mathrm{x},-\mathrm{y}, 1+\mathrm{z}$ & $3.943(4)$ & $0.0(3)$ & $-3.379(2)$ & $3.378(2)$ & 2.034 \\
\hline $\operatorname{Cg}(2)$ & $\mathrm{Cg}(2)$ & $\mathrm{x}, \mathrm{y},-1+\mathrm{z}$ & $3.943(4)$ & $0.0(3)$ & $3.378(2)$ & $-3.379(2)$ & 2.034 \\
\hline $\operatorname{Cg}(2)$ & $\operatorname{Cg}(2)$ & $\mathrm{x}, \mathrm{y}, 1+\mathrm{z}$ & $3.943(4)$ & $0.0(3)$ & $-3.379(2)$ & $3.378(2)$ & 2.034 \\
\hline $\operatorname{Cg}(2)$ & $\mathrm{Cg}(2)$ & $\mathrm{x},-\mathrm{y},-1+\mathrm{z}$ & $4.357(3)$ & $32.6(3)$ & $2.858(2)$ & $-3.898(2)$ & - \\
\hline $\operatorname{Cg}(2)$ & $\operatorname{Cg}(2)$ & $\mathrm{x},-\mathrm{y}, 1+\mathrm{z}$ & $4.357(3)$ & $32.6(3)$ & $-3.898(2)$ & $2.858(2)$ & - \\
\hline
\end{tabular}




\section{Complex 17}

CCDC number: 1879143

Relevant ring planes: $\mathrm{Cg}(1)=\mathrm{N} 1-\mathrm{N} 2-\mathrm{C} 3-\mathrm{C} 2-\mathrm{C} 1 ; \mathrm{Cg}(2)=\mathrm{N} 1-\mathrm{N} 2-\mathrm{C} 4-\mathrm{C} 5-\mathrm{C} 6$;

$\mathrm{Cg}(3)=\mathrm{N} 3-\mathrm{N} 4-\mathrm{C} 13-\mathrm{C} 12-\mathrm{C} 11 ; \mathrm{Cg}(4)=\mathrm{N} 3-\mathrm{N} 4-\mathrm{C} 14-\mathrm{C} 15-\mathrm{C} 16$

Note: The values in this table were calculated using CrystalMaker v. 10.3.3, instead of Platon.

\begin{tabular}{|c|c|c|c|c|c|}
\hline $\operatorname{Cg}(i)$ & $\operatorname{Cg}(j)$ & Symmetry $\operatorname{Cg}(j)$ & $\operatorname{Cg}-\mathrm{Cg}(\AA)$ & Cg(i)_perp $(\AA)$ & Cg(j)_perp $(\AA)$ \\
\hline $\operatorname{Cg}(1)$ & $\mathrm{Cg}(3)$ & $2-x, 1-y, 1-z$ & 3.943 & 2.7991 & 3.6763 \\
\hline $\mathrm{Cg}(1)$ & $\mathrm{Cg}(4)$ & $2-x, 1-y, 1-z$ & 3.396 & 3.3669 & 3.2965 \\
\hline $\mathrm{Cg}(2)$ & $\operatorname{Cg}(3)$ & $2-x, 1-y, 1-z$ & 3.412 & 3.2783 & 3.1924 \\
\hline $\mathrm{Cg}(2)$ & $\mathrm{Cg}(4)$ & $2-x, 1-y, 1-z$ & 3.728 & 2.9110 & 3.6092 \\
\hline $\mathrm{Cg}(3)$ & $\operatorname{Cg}(1)$ & $\mathrm{y}, \mathrm{x}, \mathrm{z}+1$ & 4.262 & 3.9327 & 2.9311 \\
\hline $\mathrm{Cg}(4)$ & $\operatorname{Cg}(1)$ & $\mathrm{y}, \mathrm{x}, \mathrm{z}+1$ & 3.507 & 3.2068 & 3.2405 \\
\hline $\mathrm{Cg}(3)$ & $\mathrm{Cg}(2)$ & $\mathrm{y}, \mathrm{x}, \mathrm{z}+1$ & 3.601 & 3.5394 & 3.4588 \\
\hline $\mathrm{Cg}(4)$ & $\operatorname{Cg}(2)$ & $\mathrm{y}, \mathrm{x}, \mathrm{z}+1$ & 3.657 & 3.5924 & 3.1278 \\
\hline
\end{tabular}

